\title{
Intrinsic Broadening of the Transverse Momentum Spectra in Ultrarelativistic Heavy-Ion Collisions?
}

\author{
Larry McLerran ${ }^{a}$ and Jürgen Schaffner-Bielich ${ }^{b}$ \\ ${ }^{a}$ Department of Physics, Brookhaven National Laboratory, Upton, NY 11973-5000, USA \\ ${ }^{b}$ RIKEN BNL Research Center, Brookhaven National Laboratory, Upton, NY 11973-5000, USA
}

(December 6, 2018)

\begin{abstract}
The mean transverse momentum in high energy nuclear collisions is studied. The formation of a Color Glass Condensate results in an increase of the square of the mean transverse momentum with the charged multiplicity per unit rapidity and unit transverse area. This prediction is in agreement with the presently available data, in particular with the heavy-ion data, lending support to the notion that the transverse momentum spectra in ultrarelativistic collisions of heavy nuclei might be controlled by intrinsic $p_{T}$ broadening and not by hadronic rescattering, i.e. transverse flow.
\end{abstract}


The initial gluon distribution in high-energy nuclear collisions can be described by the formation of a so called Color Glass Condensate (CGC) [1] 3]. The gluon multiplicity per unit rapidity and transverse area has been shown to depend on the initial gluon density which saturates at very high energies 1 国. This saturation should be observable in the mean transverse momentum spectra which should scale then like the square root of the charged multiplicity per unit rapidity and unit transverse area, hence:

$$
\left\langle p_{T}\right\rangle^{2} \sim \frac{1}{\pi R^{2}} \frac{d N_{c}}{d y}
$$

by dimensional arguments. In this paper, we are going to confront this prediction with presently available experimental data. As we will outline in the following, we find that the above relation eq. (1) is fulfilled by both, pp or $\mathrm{p} \overline{\mathrm{p}}$ and heavy-ion data. This observation seems to suggest that the transverse momentum broadening seen in elementary and heavyion collisions results from the same underlying physics, namely the intrinsically generated $p_{T}$ broadening in the partonic phase, i.e. from the CGC. This implies that transverse flow from rescattering in heavy-ion collisions in the hadronic phase might be small compared to the transverse push initially generated in the partonic phase.

The study of the particle transverse momentum as a function of multiplicity is interesting as it might reveal features of a phase transition to a quark-gluon plasma [9]. The increase of the mean $p_{T}$ with the charged multiplicity is a well known experimental fact for highenergy electron and proton-antiproton collisions (see e.g. [10,[1] and references therein). Mass identified particle production were measured at the Tevatron for p $\bar{p}$ collisions at up to $\sqrt{s}=1.8 \mathrm{TeV}$ by the E735 collaboration [12,13]. The data is shown in fig. 1. It is apparent from the figure, that the increase of the mean $p_{T}$ depends on the mass of the hadron and is stronger for heavier particles. We have also plotted the results of two-parameter fits using the scaling law eq. (1) by the solid curves. The two parameters are the coefficient of $\sqrt{d N_{c} / d y}$ and the value of $p_{T}$ as the multiplicity shrinks to zero. We note that the agreement with the data is quite reasonable. The mean $p_{T}$ seems to follow the saturation momentum of the gluon distribution function resulting in a square root dependence with the multiplicity density. The onset of the curve at small mean $p_{T}$ is due to soft physics. The difference in the slope parameter for pions, kaons, antiprotons will be discussed later.

We have also checked numerically that the more accurate data for all negatively charged

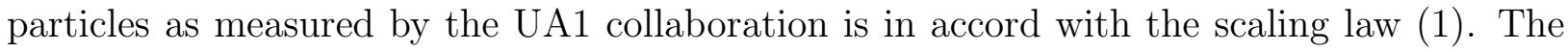
slope is slightly larger than for the pions in fig. 1 d due to the small admixture of negatively charged kaons and antiprotons. The increase of the mean $p_{T}$ with multiplicity for $\mathrm{p} \overline{\mathrm{p}}$ for the different hadrons has been also reproduced by more sophisticated partonic models, like the HIJING model [14], the quark-gluon string model [15], and the dual parton model [16]. Radial flow has been also suggested as a possible explanation [17], but it is at variance with the small source sizes determined from two-pion correlations [18].

Next, we test our model for relativistic (heavy-)ion collisions. Presently published data with the highest available bombarding energy is from the ISR collider at CERN for light ions up to $\alpha \alpha$ collisions [19] An enhancement of the slope of the mean $p_{T}$ with the multiplicity density is observed. At the same bombarding energy of $\sqrt{s}=31 \mathrm{GeV}$, the increase amounts to about $30 \%$ for $\alpha \alpha$ collisions compared to dd collisions, which is about $30 \%$ larger than that for pp collisions. According to (1), the increase of the slope of the mean $p_{T}$ squared 


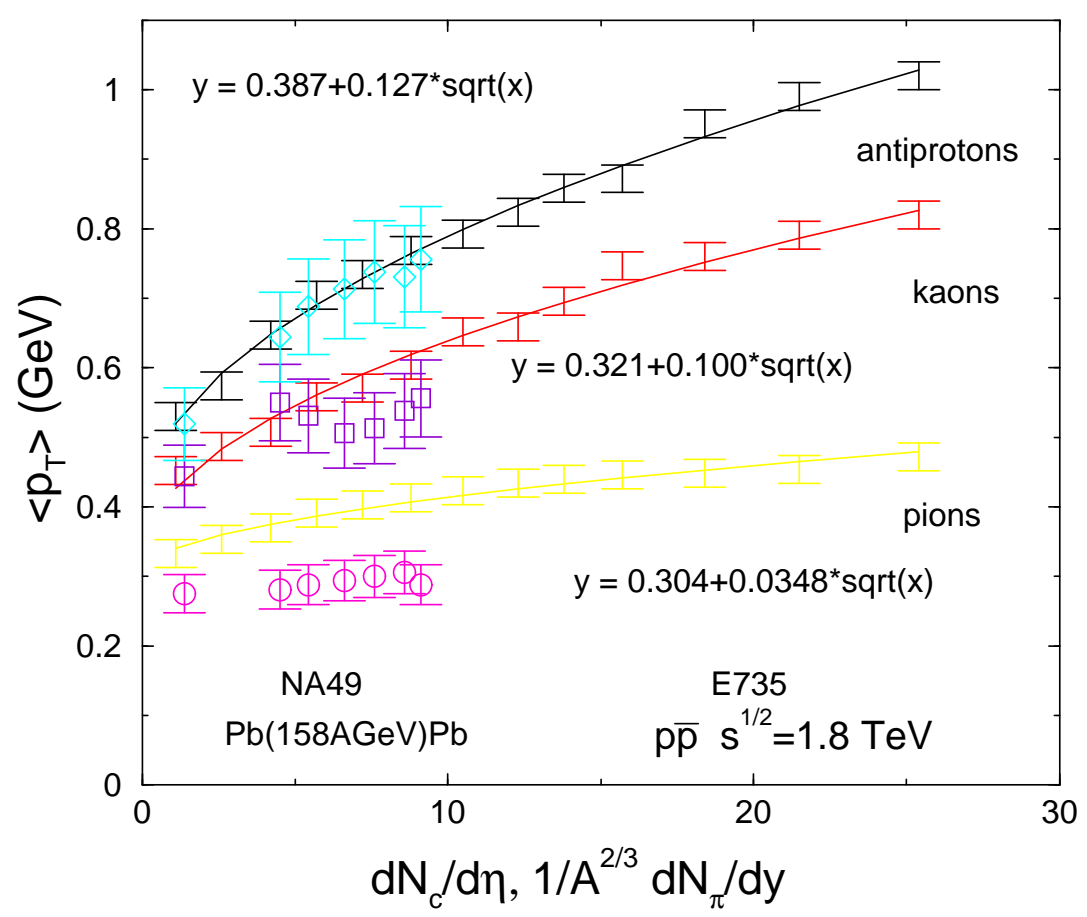

FIG. 1. The mean $p_{T}$ as a function of the charged multiplicity per unit rapidity and area. Shown are the data points for $\mathrm{p} \overline{\mathrm{p}}$ collisions at $\sqrt{s}=1.8 \mathrm{TeV}$ from E735 at the Tevatron as taken from [13], and heavy-ion data for pp and PbPb collisions at $158 \mathrm{AGeV}$ from NA49 at the SPS as extracted from [20]. The solid lines are fits through the Tevatron data using the scaling law of the CGC.

should scale like the unit transverse area, i.e. with $A^{2 / 3}$. More precisely, it would scale like $r_{p}^{2} /\left(r_{0}^{2} A^{2 / 3}\right)$ where $r_{p}$ is the proton radius and $r_{0} \approx 1.1$. The mean $p_{T}$ will increase then by about $2^{1 / 3}$, i.e. by $26 \%$, when going from pp to dd to $\alpha \alpha$ in excellent agreement with the observation made at ISR [19].

Concerning truly heavy-ion collisions, the first events from Brookhaven's Relativistic Heavy-Ion Collider (RHIC) for AuAu collisions at $\sqrt{s}=130 \mathrm{AGeV}$ are presently under investigation [21]. The NA49 experiment at CERN's SPS has published multiplicity and mean $p_{T}$ distributions as a function of the centrality for $\mathrm{PbPb}$ collisions at midrapidity [20]. We extract the charged particle multiplicity per unit rapidity from the pion multiplicity and by setting $d N / d y=160$ for the most central bin. The charged multiplicity density per unit transverse area is then given by dividing it with $A^{2 / 3}$ (setting the proton radius to $r_{p}=1$ $\mathrm{fm}$ would give an additional shift in the scale down by $\left(r_{p} / r_{0}\right)^{2}$, i.e. by $\left.20 \%\right)$. Systematic error bars for the mean $p_{T}$ have not been given in [20]. We adopt an ad hoc value of $10 \%$ for the systematic error [22]. The resulting points are plotted in fig. 1. We note that the results for the protons and kaons are then within the error bars on top of the data from the Tevatron and the corresponding fit for a CGC. The results for the mean $p_{T}$ for the pions are somewhat lower though.

However, this difference is due to the moderate dependence of the mean $p_{T}$ on the bombarding energy (see [10,13] and references therein). For energies comparable to the SPS the mean transverse momentum averaged over multiplicity $\left\langle p_{T}\right\rangle$ is about $0.33 \mathrm{GeV}$ for pions for $\mathrm{p} \overline{\mathrm{p}}$ collisions and increases to $0.38 \mathrm{GeV}$ at $\sqrt{s}=1.8 \mathrm{TeV}$. If the points for the pions from 
NA49 are shifted accordingly, they are consistent with our fit to the Tevatron data points. A similar shift for the kaons points does not change the agreement with the scaling curve. The proton points are then slightly higher than our scaling curve fitted to the Tevatron data, but they are consistent with the Tevatron data points within the error bars. The slight energy dependence of $\left\langle p_{T}\right\rangle$ can be explained by a slight increase of the saturation momentum with $\sqrt{s}$. Assuming a power law behavior [23], we find that the energy dependence of $\left\langle p_{T}\right\rangle$ for charged particles as measured by the UA1 collaboration goes like $\sim E^{\delta} \llbracket 10$ with $\delta=0.08$. Note, that a logarithmic dependence is also in accord with the data [10]. The increase with energy is considerably larger for heavier hadrons as seen by the E735 collaboration [13], so that for protons we get $\delta=0.19$ instead. We remark, that the data seems to indicate that there is an threshold effect, an onset of the increase of the transverse momentum at an energy around $\sqrt{s}=100-200 \mathrm{GeV}$ which is right in the region accessible at RHIC. The gluon distribution function starts to turn on at $x<0.01$. This is the region where the rise in $p_{T}$ should begin. In heavy-ion reactions the start of the rise appears at smaller energies which might be due to the increase of the gluon density in AA collisions compared to pp collisions. The onset of gluon saturation might be visible by studying the mean transverse momentum for $\mathrm{pA}$ and light AB systems at the SPS. At a certain size of the colliding system, the $\left\langle p_{T}\right\rangle^{2}$ dependence should then increase with the multiplicity per unit transverse area. There will be also a moderate enhancement of $\left\langle p_{T}\right\rangle$ compared to pp collisions due to the increase of the saturation momentum $Q_{s}^{2}$ with $A^{1 / 3}$. The A dependence of the color-glass condensate for pA and light systems has been studied in detail in [24].

In conclusion, there seems to be a dramatic correspondence of the mean $p_{T}$ appropriately scaled by the transverse unit area for heavy-ion collisions and $\mathrm{p} \overline{\mathrm{p}}$ collisions at much larger energies. The origin of this behavior is lying in the saturation of the initial gluon transverse momentum distribution. This observation, if taken seriously, leads to a drastic conclusion: it is possible that the transverse momentum distribution of hadrons in heavy-ion collisions is mainly controlled by the initial momentum distributions of gluons and not by effects from hadronic rescattering. The good agreement between the two curves from $\mathrm{p} \overline{\mathrm{p}}$ and heavy-ion collisions for the mean $p_{T}$ does not leave room for a significant transverse expansion via a collective transverse flow. We note, that a random walk model can also describe the $p_{T}$ broadening in heavy-ion collisions without transverse flow [25] (see also [26] for a critical discussion).

What we left open is the question why heavier particles have larger mean $p_{T}$ in $\mathrm{p} \overline{\mathrm{p}}$ and in heavy-ion collisions. A possible explanation can be provided by the coalescence model for the formation of hadrons at the confinement phase transition. Of course, the formation of hadrons is an extremely complex process which requires a detailed dynamical approach. With this in mind, let us state the following. Naively, the mean $p_{T}$ of a hadron will scale with the number of quarks in the coalescence model as the quark momenta are added up to form a hadron. The mean $p_{T}$ for protons should then be $3 / 2$ times larger than that for pions. The strange quark will take a special role in this picture as the associated production from gluons needs to overcome the mass threshold. After coalescence, many pions will come from subsequent resonance decays, e.g. from the $\rho$ meson. The $\rho$ meson picks up 2 times the intrinsic $p_{T}$, but decays to two pions with about half that $p_{T}$. Hence, the combination of these effects results in a $p_{T}$ ratio between $3 / 2$ and 3 . The momentum spectra of kaons on the other hand are not affected by this resonance effect and end up with about $2 / 3$ times the 
proton mean $p_{T}$. Indeed, the mean $p_{T}$ for protons compared to pions shows an increase by about 1.5-2.0 over a wide range of energy (see e.g. the data compiled in [13]), the kaon mean $p_{T}$ being between the mean $p_{T}$ for pions and protons. Also, the slope parameters measured for pions and protons in $\mathrm{PbPb}$ collisions has been extracted to be around $190 \mathrm{MeV}$ and 300 $\mathrm{MeV}$ [27], respectively, reflecting a similar increase in the mean $p_{T}$.

We conclude with a brief comment about a possible test of this picture. The physical quantity which is computed from the Color Glass Condensate is the phase space distribution

$$
\frac{1}{\pi R^{2}} \frac{d N}{d y d^{2} p_{T}}
$$

On dimensional grounds for equal size nuclear collision at some fixed impact parameter

$$
\frac{1}{\sigma} \frac{d N}{d y d^{2} p_{T}}=F\left(Q_{s}^{2} / p_{T}^{2}\right)
$$

In this equation, $Q_{s}^{2}$ is the saturation momentum squared which characterizes the Color Glass Condensate at the impact parameter for the collision. An attempt to extract this saturation momentum has been done by Kharzeev and Nardi [8]. The cross section $\sigma$ is that for a collision to occur at the impact parameter at hand. This formula predicts that the $p_{T}$ distributions at different impact parameters can be rescaled into one another. If true, it is a remarkable verification of the approximate scale-invariance of the Color Glass Condensate.

\section{ACKNOWLEDGMENTS}

J.S.B. thanks the Institut für Theoretische Physik at the University of Frankfurt, where parts of this work were completed, for their warm hospitality, and RIKEN, BNL, and the U.S. Department of Energy for providing the facilities essential for the completion of this work. This manuscript has been authorized with the U.S. Department of Energy under Contract No. DE-AC02-98CH10886. 


\section{REFERENCES}

[1] L. McLerran and R. Venugopalan, Phys. Rev. D49, 2233 (1994); ibid 3352 (1994); Y. Kovchegov, Phys. Rev. D54, 5463 (1996).

[2] J. Jalilian-Marian, A. Kovner, L. McLerran, and H. Weigert, Phys. Rev. D55, 5414 (1997); J. Jalilian-Marian, A. Kovner, A. Leonidov, and H. Weigert, Nucl. Phys. B504, 415 (1997); Phys. Rev. D59, 014014 (1999).

[3] E. Iancu, A. Leonidov, and L. McLerran, hep-ph/0011241 (2000).

[4] L. V. Gribov, E. M. Levin, and M. G. Ryskin, Phys. Rep. 100, 1 (1983).

[5] A. H. Mueller and J. W. Qiu, Nucl. Phys. B268, 427 (1986).

[6] A. Kovner, L. McLerran, and H. Weigert, Phys. Rev. D52, 6231 (1995); ibid 3809 (1995).

[7] A. Krasnitz and R. Venugopalan, Phys. Rev. Lett. 86, 1717 (2001); W. Pöschl and B. Müller, Comput. Phys. Commun. 125, 282 (2000).

[8] D. Kharzeev and M. Nardi, Phys. Lett. B 507, 121 (2001).

[9] L. van Hove, Phys. Lett. 118B, 138 (1982).

[10] UA1 collaboration, C. Albajar et al., Nucl. Phys. B335, 261 (1990).

[11] E735 collaboration, T. Alexopoulos et al., Phys. Rev. Lett. 60, 1622 (1988).

[12] E735 collaboration, T. Alexopoulos et al., Phys. Rev. Lett. 64, 991 (1990).

[13] E735 collaboration, T. Alexopoulos et al., Phys. Rev. D48, 984 (1993).

[14] X.-N. Wang and M. Gyulassy, Phys. Rev. D45, 844 (1992).

[15] N. S. Amelin, E. F. Staubo, and L. P. Csernai, Phys. Rev. D46, 4873 (1992).

[16] F. W. Bopp, R. Engel, D. Pertermann, and J. Ranft, Phys. Rev. D49, 3236 (1994).

[17] P. Levai and B. Müller, Phys. Rev. Lett. 67, 1519 (1991).

[18] E735 collaboration, T. Alexopoulos et al., Phys. Rev. D48, 1931 (1993).

[19] A. Breakstone et al., Phys. Lett. B183, 227 (1987).

[20] NA49 collaboration, J. Bächler et al., Nucl. Phys. A661, 45 (1999).

[21] J. Velkovska [PHENIX collaboration], nucl-ex/0105012 (2001); J. W. Harris and M. Calderon [STAR collaboration], talks given at Quark Matter 2001, Stony Brook, New York, 2001; Barbara Jacak, private communication.

[22] Ferenc Siklér, private communication.

[23] Y. V. Kovchegov, Phys. Rev. D61, 074018 (2000).

[24] A. Dumitru and L. McLerran, hep-ph/0105268 (2001).

[25] A. Leonidov, M. Nardi, and H. Satz, Z. Phys. C74, 535 (1997).

[26] H. Satz, Nucl. Phys. B (Proc. Suppl.) 94, 204 (2001).

[27] NA49 collaboration, T. Wienold et al., Nucl. Phys. A610, 76c (1996). 